\title{
ESTUDIO ESTADÍSTICO DE APLICACIÓN DE LAS FÓRMULAS PROPUESTAS PARA PROYECTAR LA RESISTENCIA DEL HORMIGÓN
}

\author{
M. I. SCHIERLOH, R. F. SOUCHETTI, L. D. DEUSICH \\ Grupo de Investigación en Rehabilitación de Estructuras (G.I.R.E.), Departamento de Ingeniería Civil, Facultad Regional \\ Concepción del Uruguay, Universidad Tecnológica Nacional, Ingeniero Pereira 676, Concepción del Uruguay, Entre \\ Ríos, Argentina.
}

\begin{abstract}
RESUMEN
Las fórmulas propuestas para proyectar la evolución de la resistencia del hormigón en el tiempo, fueron desarrolladas a partir de 30 series de probetas. El presente trabajo muestra, los resultados alcanzados a partir de un estudio estadístico desarrollado, para obtener coeficientes que permitan contemplar la variación en los resultados al aplicar dichas fórmulas, para predecir la resistencia especificada del hormigón a 28 días, usando un menor número de muestras. Se tuvieron en cuenta, las prescripciones de la reglamentación vigente en Argentina, el cual especifica un mínimo de 3 probetas por pastón, alcanzándose valores para dichos coeficientes que al aplicarlos garantizaron que la tensión será mayor en un $95 \%$ de los resultados, a la especificada para los 28 días.
\end{abstract}

Palabras clave: Resistencia del hormigón; evolución de la resistencia en el tiempo; coeficientes de aproximación.

\begin{abstract}
The formulas proposed to project the evolution of concrete strength over time, were developed from 30 series of specimens. The present work shows, the results obtained from a statistical study developed, to obtain coefficients that allow to contemplate the variation in the results when applying said formulas, to predict the specified resistance of the concrete to 28 days, using a smaller number of samples. The prescriptions of the regulations in force in Argentina were taken into account, which specifies a minimum of 3 specimens per paston, reaching values for these coefficients that when applied guaranteed that the tension will be greater by $95 \%$ of the results, as specified for the 28 days.
\end{abstract}

Keywords: Concrete strength; evolution of resistance over time; approximation coefficients

\section{RESUMO}

As fórmulas propostas para projetar a evolução da resistência do concreto ao longo do tempo foram desenvolvidas a partir de 30 séries de espécimes. O presente trabalho mostra, os resultados obtidos a partir de um estudo estatístico desenvolvido, para obter coeficientes que permitem contemplar a variação dos resultados na aplicação das referidas fórmulas, para prever a resistência especificada do concreto a 28 dias, utilizando um menor número de amostras. Foram consideradas as prescrições dos regulamentos vigentes na Argentina, que especificam um mínimo de 3 exemplares por paston, atingindo valores para esses coeficientes que quando aplicados garantiram que a tensão será maior em 95\% dos resultados, conforme especificado para os 28 dias.

Palavras-chave: Força do concreto; evolução da resistência ao longo do tempo; coeficientes de aproximação. 


\section{INTRODUCCIÓN}

Ha sido una práctica muy generalizada en Argentina, usar la fórmula de Ros, que fue establecida para cementos suizos y para hormigones elaborados y curados en laboratorio (Fava, 1948).

Otros investigadores, europeos y americanos (Bach, Graff, Hummel, etc.), han definido diversas fórmulas también, para determinar la resistencia del hormigón a compresión en una edad avanzada, partiendo de ensayos a corto plazo (Hummel, 1966). En todos los casos las fórmulas dependen entre otras variables, del tipo de cemento usado. Cada investigador ha procurado hacer intervenir en sus ecuaciones las múltiples variables influyentes en la calidad, partiendo de la relación agua-material cementicio, calidad y tipo de cemento, tipo de áridos, temperatura ambiente, medios tecnológicos de producción etc.

Algunos países como España, incluye en su instrucción EHE (Instrucción española del Hormigón Estructural), en el art.30.4b, una tabla con valores estimativos de la resistencia a la compresión a la edad de "j" días en relación con la resistencia a los 28 días. (IEHE, 2008). En la actualidad, la composición de los cementos argentinos, han variado mucho, siendo aún mayor el desfasaje entre las curvas de Ros y otros investigadores europeos y americanos, con las que surgen usando los cementos argentinos (Hummel, 1966). Debido a ello, Vialidad Nacional Argentina, ha dejado de validar retrotraer o proyectar los valores de resistencia con fórmulas por ejemplo (VNA, 2015).

El requerimiento de predecir a edades tempranas la resistencia característica o especificada del hormigón a los 28 días, es una necesidad permanente en el ámbito de la construcción, sobre todo en obras de gran envergadura, donde poder proyectar en algunos casos, y verificar en otros, dicha resistencia, es fundamental para el avance de los trabajos.

Las normas para proyectar las estructuras de hormigón se basan, en cuanto a calidad se refiere, en los resultados del ensayo a la compresión simple de probetas preparadas y curadas en condiciones normalizadas a los 28 días de edad. Este período, muchas veces genera dificultades pues sus resultados se conocen cuando el avance de la obra hace muy complejo y costoso su reemplazo, en el caso de no cumplir con las condiciones prefijadas.

El método denominado natural, para predecir la resistencia característica o especificada del hormigón, consiste en realizar pastones de hormigón, para un tipo de cemento en particular, colocarlos en moldes, curarlos en forma normalizada a temperatura controlada y ensayar probetas ejecutadas de un mismo pastón a diferentes edades y a los 28 días. Posteriormente se relacionan ambos valores a través de ecuaciones para lograr predecir la resistencia característica o especificada, lo cual conlleva ciertos rangos de error, dependiendo de la edad en que se ha ensayo el hormigón.

En este trabajo se presenta el estudio estadístico de los resultados obtenidos, a partir de la aplicación de las fórmulas propuestas, en el trabajo " Estudio sobre variación de la resistencia a compresión en el tiempo, para hormigones utilizando cementos argentinos y áridos de la zona de Concepción del Uruguay - Entre Ríos"(Schierloh et.al., 2018), para proyectar la resistencia del hormigón obtenida a diferentes edades, a los 28 días especificado por norma.

\section{PROCEDIMIENTO}

Para llevar a cabo el estudio propuesto se trabajando fundamentalmente desde el campo experimental y con los resultados obtenidos a partir de allí, aplicando la teoría matemática, se utilizaron sistemas estadísticos y metodología numérico-computacional.

Se realizaron un total de 30 series de probetas por cada tipo de cemento, se trabajó con cemento portland compuesto CPC40 (Lomanegra) y filerizado CPF40 (Avellaneda). Cada una de las series estuvo compuesta por catorce probetas de hormigón. De estas, dos se usaron para la rotura a tracción por compresión diametral Las restantes doce, se usaron para determinar los valores de resistencia a 
compresión a los 3, 7, 14, 28, 45 y 60 días, haciendo roturas dobles y tomando la media aritmética de los valores de rotura a cada edad (Schierloh et.al., 2018).

Por lo tanto, para cada cemento, se elaboraron y ensayaron cuatrocientas veinte (420) probetas. De esta manera, los valores de resistencia a compresión determinados a los 3, 7, 14, 28, 45 y 60 días, resultaron del promedio de dos resultados de ensayo, teniendo la precaución de cumplir con la normativa que especifica no superar el valor de rotura de cada probeta individualmente, el 15\% de la media aritmética de ambos valores.

El Hormigón fue dosificado según las recomendaciones del reglamento CIRSOC 201:05 (C.I.R.S.O.C. 201, 2005), inicialmente clase H-20 para una relación agua cemento de 0,5 , asentamiento de $8 \mathrm{~cm}$.

Las fórmulas para proyectar la resistencia del hormigón fueron determinadas según el procedimiento encontrado al desarrollar el trabajo referenciado (Schierloh et.al., 2018) y patentado bajo el código AR111556A1, publicado en el boletín oficial de Patentes Argentina el 24 de julio de 2019.

\section{ANALISIS ESTADISTICO PARA MEZCLAS CON CEMENTO CPC 40}

\subsection{Estadística individual}

Las fórmulas encontradas para ambos cementos resultaron del proceso de ensayar treinta (30) series de probetas para cada caso, según lo requieren las normas actuales.

Para cantidades de series ensayadas mayores a treinta (30), la desviación de los valores de ensayo obtenidos, respecto de los valores teóricos que deberían dar, aplicando las fórmulas se hacen cada vez más despreciable, a medida que ampliamos el número de ensayos.

Para cantidades de series ensayadas menores a treinta (30), la desviación que podemos encontrar entre los valores reales de rotura y los valores teóricos que debería dar aplicando las fórmulas, se incrementa a medida que nos acercamos a valores individuales de ensayos de probetas.

La Tabla 1, resume este concepto para el caso del cemento CPC40.

La columna tres "Rotura Compresión Mpa", indica los valores de roturas de cada serie, a los diferentes días de ensayo, expresado en megapascales.

La columna cuatro " $\sigma 28$ según G.I.R.E. Mpa", indica los valores que debería alcanzar cada serie a los veintiocho días, según los días de rotura.

La columna cinco "Diferencia entre $\sigma 28$ G.I.R.E. y ensayo - Mpa", representa la diferencia en megapascales entre el valor teórico calculado con la fórmula propuesta para CPC40, a los 28 días y el valor real de rotura a los 28 días.

Los valore negativos, indican que los valores de ensayo son menores a los valores teóricos calculados con la fórmula y viceversa.

La columna seis "Diferencia en porcentaje", representa la diferencia o desviación en porcentaje, entre el valor teórico de la formula y el valor obtenido por ensayos de probetas.

Los valores negativos, muestran que los valores de rotura resultaron menores a los teóricos calculados con la formula y viceversa.

Si por ejemplo se toma la primera fila de la serie I, se aprecia que, a los 3 días, la probeta rompió a $16,84 \mathrm{Mpa}$. Se recuerda que esta rotura representa el valor promedio de dos ensayos, tal cual lo exigen como mínimo las normas actuales.

Si se aplica la formula, a los 28 días debería romper a 32,46 Mpa.

La realidad mostró, que a los 28 días el valor de rotura fue de 27,73 Mpa, habiendo una diferencia de 4,59 Mpa., que, expresado en porcentaje respecto del valor teórico de resultado de aplicar la fórmula, representa un $-14,19 \%$

Lo cual indicó, que individualmente a los 3 días, el valor de rotura a los 28 días fue un 14,19 menor al valor teórico que debería dar aplicando la formula. 
Tabla 1. Desviación de valores comparativos individuales, entre formula G.I.R.E. y los valores de rotura para mezclas con cemento CPC 40.

\begin{tabular}{|c|c|c|c|c|c|}
\hline serie & $\begin{array}{c}\text { Días de } \\
\text { rotura }\end{array}$ & $\begin{array}{c}\text { Rotura } \\
\text { compresión } \\
\text { Mpa }\end{array}$ & $\begin{array}{c}\sigma 28 \text { dias } \\
\text { según g.i.r.e. } \\
\text { Mpa }\end{array}$ & $\begin{array}{c}\text { Diferencia } \\
\text { con } \sigma 28 \text { días } \\
\text { según ensayo - } \\
\text { Mpa }\end{array}$ & $\begin{array}{c}\text { Diferencia en } \\
\text { porcentaje }\end{array}$ \\
\hline \multirow{6}{*}{ I } & 3 & 16,84 & 32,46 & $-4,73$ & $-14,58$ \\
\hline & 7 & 20,94 & 27,06 & 0,67 & 2,48 \\
\hline & 14 & 24,63 & 26,97 & 0,76 & 2,81 \\
\hline & 28 & 27,73 & 27,63 & 0,10 & 0,36 \\
\hline & 45 & 29,73 & 28,46 & $-0,73$ & $-2,56$ \\
\hline & 60 & 31,27 & 29,40 & $-1,67$ & $-5,68$ \\
\hline \multirow{6}{*}{ II } & 3 & 17,88 & 34,48 & $-6,80$ & $-19,71$ \\
\hline & 7 & 22,02 & 28,44 & $-0,76$ & $-2,68$ \\
\hline & 14 & 25,58 & 28,01 & $-0,33$ & $-1,19$ \\
\hline & 28 & 27,68 & 27,58 & 0,10 & 0,36 \\
\hline & 45 & 29,63 & 28,37 & $-0,69$ & $-2,42$ \\
\hline & 60 & 30,12 & 28,32 & $-0,64$ & $-2,26$ \\
\hline \multirow{6}{*}{ III } & 3 & 17,91 & 34,53 & $-6,87$ & $-19,89$ \\
\hline & 7 & 22,74 & 29,38 & $-1,72$ & $-5,86$ \\
\hline & 14 & 25,64 & 28,08 & $-0,42$ & $-1,49$ \\
\hline & 28 & 27,66 & 27,56 & 0,10 & 0,36 \\
\hline & 45 & 28,38 & 27,17 & 0,49 & 1,82 \\
\hline & 60 & 29,28 & 27,54 & 0,12 & 0,45 \\
\hline \multirow{6}{*}{ IV } & 3 & 20,05 & 38,66 & $-9,61$ & $-24,86$ \\
\hline & 7 & 25,83 & 33,37 & $-4,32$ & $-12,95$ \\
\hline & 14 & 28,86 & 31,61 & $-2,56$ & $-8,09$ \\
\hline & 28 & 29,05 & 28,94 & 0,11 & 0,37 \\
\hline & 45 & 29,49 & 28,23 & 0,82 & 2,91 \\
\hline & 60 & 30,19 & 28,39 & 0,66 & 2,33 \\
\hline \multirow{6}{*}{ V } & 3 & 14,07 & 27,13 & $-2,29$ & $-8,44$ \\
\hline & 7 & 20,66 & 26,69 & $-1,85$ & $-6,94$ \\
\hline & 14 & 23,05 & 25,24 & $-0,40$ & $-1,58$ \\
\hline & 28 & 24,84 & 24,75 & 0,09 & 0,37 \\
\hline & 45 & 25,67 & 24,58 & 0,26 & 1,07 \\
\hline & 60 & 26,14 & 24,58 & 0,26 & 1,05 \\
\hline \multirow{6}{*}{ VI } & 3 & 16,16 & 31,17 & $-4,38$ & $-14,04$ \\
\hline & 7 & 21,72 & 28,06 & $-1,27$ & $-4,51$ \\
\hline & 14 & 24,05 & 26,33 & 0,46 & 1,73 \\
\hline & 28 & 25,66 & 25,57 & 1,22 & 4,76 \\
\hline & 45 & 26,81 & 25,67 & 1,12 & 4,37 \\
\hline & 60 & 27,40 & 25,77 & 1,02 & 3,97 \\
\hline
\end{tabular}


VICC194 - CONPAT 2019, Tuxtla Gutiérrez, Chiapas, México

\begin{tabular}{|c|c|c|c|c|c|}
\hline \multirow{6}{*}{ VII } & 3 & 20,70 & 39,91 & $-13,12$ & $-32,88$ \\
\hline & 7 & 23,94 & 30,93 & $-4,14$ & $-13,39$ \\
\hline & 14 & 25,53 & 27,96 & $-1,17$ & $-4,18$ \\
\hline & 28 & 26,79 & 26,70 & 0,09 & 0,35 \\
\hline & 45 & 28,05 & 26,85 & $-0,06$ & $-0,23$ \\
\hline & 60 & 29,06 & 27,33 & $-0,54$ & $-1,97$ \\
\hline \multirow{6}{*}{ VIII } & 3 & 17,81 & 34,35 & $-7,45$ & $-21,68$ \\
\hline & 7 & 24,96 & 32,24 & $-5,34$ & $-16,58$ \\
\hline & 14 & 25,66 & 28,10 & $-1,20$ & $-4,28$ \\
\hline & 28 & 26,90 & 26,80 & 0,10 & 0,37 \\
\hline & 45 & 27,32 & 26,16 & 0,74 & 2,84 \\
\hline & 60 & 27,78 & 26,12 & 0,78 & 2,98 \\
\hline \multirow{6}{*}{ IX } & 3 & 16,14 & 31,12 & $-5,62$ & $-18,07$ \\
\hline & 7 & 22,14 & 28,60 & $-3,10$ & $-10,84$ \\
\hline & 14 & 23,93 & 26,20 & $-0,70$ & $-2,67$ \\
\hline & 28 & 25,50 & 25,41 & 0,09 & 0,36 \\
\hline & 45 & 26,99 & 25,83 & $-0,33$ & $-1,28$ \\
\hline & 60 & 28,60 & 26,89 & $-1,39$ & $-5,18$ \\
\hline \multirow{6}{*}{$X$} & 3 & 17,70 & 34,13 & $-4,38$ & $-12,84$ \\
\hline & 7 & 22,92 & 29,61 & 0,14 & 0,47 \\
\hline & 14 & 26,71 & 29,25 & 0,50 & 1,70 \\
\hline & 28 & 28,75 & 28,64 & 1,11 & 3,86 \\
\hline & 45 & 29,36 & 28,11 & 1,64 & 5,85 \\
\hline & 60 & 30,04 & 28,25 & 1,50 & 5,30 \\
\hline \multirow{6}{*}{ XI } & 3 & 17,97 & 34,65 & $-5,20$ & $-15,00$ \\
\hline & 7 & 22,94 & 29,63 & $-0,18$ & $-0,62$ \\
\hline & 14 & 26,77 & 29,32 & 0,13 & 0,44 \\
\hline & 28 & 29,45 & 29,35 & 0,10 & 0,35 \\
\hline & 45 & 30,21 & 28,91 & 0,54 & 1,85 \\
\hline & 60 & 30,54 & 28,72 & 0,73 & 2,54 \\
\hline \multirow{6}{*}{ XII } & 3 & 17,97 & 34,66 & $-3,92$ & $-11,30$ \\
\hline & 7 & 20,89 & 26,99 & 3,75 & 13,91 \\
\hline & 14 & 26,72 & 29,26 & 1,48 & 5,06 \\
\hline & 28 & 30,74 & 30,63 & 0,11 & 0,36 \\
\hline & 45 & 32,74 & 31,34 & $-0,60$ & $-1,93$ \\
\hline & 60 & 32,77 & 30,81 & $-0,07$ & $-0,23$ \\
\hline \multirow{6}{*}{ XIII } & 3 & 15,32 & 29,55 & $-1,83$ & $-6,19$ \\
\hline & 7 & 21,79 & 28,15 & $-0,43$ & $-1,52$ \\
\hline & 14 & 25,46 & 27,88 & $-0,16$ & $-0,57$ \\
\hline & 28 & 27,72 & 27,62 & 0,10 & 0,36 \\
\hline & 45 & 29,44 & 28,18 & $-0,46$ & $-1,64$ \\
\hline & 60 & 30,12 & 28,32 & $-0,60$ & $-2,14$ \\
\hline
\end{tabular}


CONPAT 2019, Tuxtla Gutiérrez, Chiapas, México - VICC194

\begin{tabular}{|c|c|c|c|c|c|}
\hline \multirow{6}{*}{ XIV } & 3 & 15,31 & 29,52 & $-2,95$ & $-9,99$ \\
\hline & 7 & 20,22 & 26,12 & 0,45 & 1,73 \\
\hline & 14 & 23,88 & 26,15 & 0,42 & 1,60 \\
\hline & 28 & 26,57 & 26,48 & 0,09 & 0,34 \\
\hline & 45 & 28,66 & 27,43 & $-0,86$ & $-3,14$ \\
\hline & 60 & 29,41 & 27,66 & $-1,09$ & $-3,93$ \\
\hline \multirow{6}{*}{$X V$} & 3 & 14,24 & 27,45 & $-0,07$ & $-0,26$ \\
\hline & 7 & 20,40 & 26,36 & 1,02 & 3,89 \\
\hline & 14 & 25,01 & 27,39 & $-0,01$ & $-0,05$ \\
\hline & 28 & 27,38 & 27,28 & 0,10 & 0,36 \\
\hline & 45 & 29,08 & 27,84 & $-0,46$ & $-1,66$ \\
\hline & 60 & 30,21 & 28,41 & $-1,03$ & $-3,62$ \\
\hline \multirow{6}{*}{ XVI } & 3 & 12,43 & 23,97 & 5,04 & 21,01 \\
\hline & 7 & 19,88 & 25,69 & 3,32 & 12,93 \\
\hline & 14 & 26,25 & 28,74 & 0,27 & 0,93 \\
\hline & 28 & 29,01 & 28,91 & 0,10 & 0,35 \\
\hline & 45 & 29,50 & 28,24 & 0,77 & 2,73 \\
\hline & 60 & 30,21 & 28,41 & 0,60 & 2,12 \\
\hline \multirow{6}{*}{ XVII } & 3 & 15,09 & 29,09 & $-2,26$ & $-7,77$ \\
\hline & 7 & 21,22 & 27,41 & $-0,58$ & $-2,12$ \\
\hline & 14 & 24,23 & 26,53 & 0,30 & 1,13 \\
\hline & 28 & 26,83 & 26,74 & 0,09 & 0,35 \\
\hline & 45 & 27,45 & 26,28 & 0,55 & 2,10 \\
\hline & 60 & 28,80 & 27,08 & $-0,25$ & $-0,93$ \\
\hline \multirow{6}{*}{ XVIII } & 3 & 13,72 & 26,46 & 3,03 & 11,47 \\
\hline & 7 & 20,41 & 26,36 & 3,13 & 11,86 \\
\hline & 14 & 25,96 & 28,43 & 1,06 & 3,74 \\
\hline & 28 & 29,49 & 29,39 & 0,10 & 0,36 \\
\hline & 45 & 30,38 & 29,08 & 0,41 & 1,41 \\
\hline & 60 & 30,87 & 29,02 & 0,47 & 1,60 \\
\hline \multirow{6}{*}{ XIX } & 3 & 16,37 & 31,57 & $-0,66$ & $-2,10$ \\
\hline & 7 & 25,16 & 32,50 & $-1,59$ & $-4,90$ \\
\hline & 14 & 28,55 & 31,27 & $-0,36$ & $-1,14$ \\
\hline & 28 & 30,91 & 30,80 & 0,11 & 0,35 \\
\hline & 45 & 31,68 & 30,32 & 0,59 & 1,94 \\
\hline & 60 & 33,26 & 31,27 & $-0,36$ & $-1,17$ \\
\hline \multirow{6}{*}{ XX } & 3 & 19,14 & 36,92 & $-5,76$ & $-15,59$ \\
\hline & 7 & 26,52 & 34,26 & $-3,10$ & $-9,05$ \\
\hline & 14 & 28,95 & 31,70 & $-0,54$ & $-1,71$ \\
\hline & 28 & 31,16 & 31,05 & 0,11 & 0,35 \\
\hline & 45 & 33,29 & 31,86 & $-0,70$ & $-2,21$ \\
\hline & 60 & 33,99 & 31,96 & $-0,80$ & $-2,52$ \\
\hline
\end{tabular}


VICC194 - CONPAT 2019, Tuxtla Gutiérrez, Chiapas, México

\begin{tabular}{|c|c|c|c|c|c|}
\hline \multirow{6}{*}{ XXI } & 3 & 14,53 & 28,02 & $-3,30$ & $-11,78$ \\
\hline & 7 & 19,63 & 25,37 & $-0,65$ & $-2,54$ \\
\hline & 14 & 22,80 & 24,97 & $-0,25$ & $-1,01$ \\
\hline & 28 & 24,72 & 24,63 & 0,09 & 0,35 \\
\hline & 45 & 26,76 & 25,61 & $-0,89$ & $-3,49$ \\
\hline & 60 & 27,27 & 25,64 & $-0,92$ & $-3,59$ \\
\hline \multirow{6}{*}{ XXII } & 3 & 16,14 & 31,12 & $-2,69$ & $-8,66$ \\
\hline & 7 & 23,60 & 30,49 & $-2,06$ & $-6,75$ \\
\hline & 14 & 26,55 & 29,08 & $-0,65$ & $-2,22$ \\
\hline & 28 & 28,43 & 28,33 & 0,10 & 0,34 \\
\hline & 45 & 29,60 & 28,34 & 0,09 & 0,33 \\
\hline & 60 & 30,18 & 28,38 & 0,05 & 0,19 \\
\hline \multirow{6}{*}{ XXIII } & 3 & 15,95 & 30,76 & $-1,95$ & $-6,34$ \\
\hline & 7 & 22,75 & 29,39 & $-0,58$ & $-1,97$ \\
\hline & 14 & 26,67 & 29,21 & $-0,40$ & $-1,37$ \\
\hline & 28 & 28,81 & 28,71 & 0,10 & 0,35 \\
\hline & 45 & 29,89 & 28,62 & 0,19 & 0,67 \\
\hline & 60 & 31,16 & 29,30 & $-0,49$ & $-1,68$ \\
\hline \multirow{6}{*}{ XXIV } & 3 & 13,33 & 25,71 & 1,08 & 4,21 \\
\hline & 7 & 19,61 & 25,33 & 1,46 & 5,77 \\
\hline & 14 & 23,04 & 25,23 & 1,56 & 6,19 \\
\hline & 28 & 26,79 & 26,69 & 0,10 & 0,37 \\
\hline & 45 & 28,50 & 27,28 & $-0,49$ & $-1,81$ \\
\hline & 60 & 29,01 & 27,28 & $-0,49$ & $-1,78$ \\
\hline \multirow{6}{*}{ XXV } & 3 & 23,30 & 44,93 & $-12,83$ & $-28,55$ \\
\hline & 7 & 26,82 & 34,66 & $-2,56$ & $-7,37$ \\
\hline & 14 & 29,21 & 31,99 & 0,11 & 0,35 \\
\hline & 28 & 32,10 & 31,99 & 0,11 & 0,34 \\
\hline & 45 & 33,43 & 32,00 & 0,10 & 0,30 \\
\hline & 60 & 34,38 & 32,33 & $-0,23$ & $-0,71$ \\
\hline \multirow{6}{*}{ XXVI } & 3 & 23,05 & 44,45 & $-11,83$ & $-26,62$ \\
\hline & 7 & 27,11 & 35,03 & $-2,41$ & $-6,88$ \\
\hline & 14 & 30,47 & 33,36 & $-0,74$ & $-2,23$ \\
\hline & 28 & 32,62 & 32,51 & 0,11 & 0,35 \\
\hline & 45 & 34,68 & 33,19 & $-0,57$ & $-1,73$ \\
\hline & 60 & 35,60 & 33,48 & $-0,86$ & $-2,56$ \\
\hline \multirow{6}{*}{ XXVII } & 3 & 14,97 & 28,87 & $-1,92$ & $-6,64$ \\
\hline & 7 & 21,88 & 28,27 & $-1,32$ & $-4,67$ \\
\hline & 14 & 25,34 & 27,75 & $-0,80$ & $-2,87$ \\
\hline & 28 & 26,95 & 26,86 & 0,09 & 0,35 \\
\hline & 45 & 28,45 & 27,24 & $-0,29$ & $-1,05$ \\
\hline & 60 & 29,41 & 27,65 & $-0,70$ & $-2,54$ \\
\hline
\end{tabular}


CONPAT 2019, Tuxtla Gutiérrez, Chiapas, México - VICC194

\begin{tabular}{|c|c|c|c|c|c|}
\hline \multirow{4}{*}{ XXVIII } & 3 & 14,29 & 27,56 & $-2,07$ & $-7,51$ \\
\cline { 2 - 6 } & 7 & 19,36 & 25,01 & 0,48 & 1,90 \\
\cline { 2 - 6 } & 14 & 23,81 & 26,07 & $-0,58$ & $-2,24$ \\
\cline { 2 - 6 } & 28 & 25,49 & 25,40 & 0,09 & 0,35 \\
\cline { 2 - 6 } & 45 & 25,99 & 24,88 & 0,61 & 2,45 \\
\hline & 60 & 26,48 & 24,90 & 0,59 & 2,37 \\
\hline \multirow{5}{*}{ XXIX } & 3 & 14,45 & 27,86 & 0,35 & 1,26 \\
\cline { 2 - 6 } & 7 & 22,34 & 28,86 & $-0,65$ & $-2,25$ \\
\cline { 2 - 6 } & 14 & 26,17 & 28,66 & $-0,45$ & $-1,56$ \\
\cline { 2 - 6 } & 28 & 28,21 & 28,11 & 0,10 & 0,34 \\
\cline { 2 - 6 } & 45 & 29,49 & 28,23 & $-0,02$ & $-0,07$ \\
\hline \multirow{4}{*}{ XXX } & 60 & 30,64 & 28,81 & $-0,60$ & $-2,08$ \\
\cline { 2 - 6 } & 3 & 15,13 & 29,18 & $-1,49$ & $-5,09$ \\
\cline { 2 - 6 } & 7 & 22,75 & 29,40 & $-1,71$ & $-5,81$ \\
\cline { 2 - 6 } & 14 & 26,44 & 28,95 & $-1,26$ & $-4,35$ \\
\cline { 2 - 6 } & 28 & 28,69 & 28,59 & $-0,90$ & $-3,14$ \\
\cline { 2 - 6 } & 45 & 30,05 & 28,77 & $-1,08$ & $-3,74$ \\
\hline
\end{tabular}

Analizando todas las series, las mayores desviaciones individuales según los días de rotura están expresados en la Tabla 2.

Analizando, por ejemplo, a los 3 días, la dispersión máxima negativa, se encuentra en la serie VII con un $-32,88 \%$ de desviación. Esto significa que esta serie rompió a los 28 días un 32,88 \% por debajo del valor teórico que debía dar aplicando la formula. La desviación máxima positiva, le corresponde a la serie XVI, con un $+21,01 \%$ de desviación. Es decir que esta serie a los tres días rompió un $21,01 \%$ arriba del valor teórico que debía dar aplicando la formula.

De todos estos valores, las desviaciones más peligrosas son las "negativas", ya que significa que, en la realidad, obtenemos valores menores a los 28 días, de los teóricos calculados.

Tabla 2. Desviaciones individuales, máximas y mínimas para mezclas con cemento CPC40.

\begin{tabular}{|c|c|c|}
\hline Días de rotura & Serie & Desviación en \%, Máxima y mínima \\
\hline \multirow{2}{*}{3} & VII & $-32,88$ \\
\cline { 2 - 3 } & XVI & 21,01 \\
\hline \multirow{2}{*}{14} & VIII & $-16,58$ \\
\cline { 2 - 3 } & XII & 13,91 \\
\hline \multirow{2}{*}{45} & IV & $-8,09$ \\
\cline { 2 - 3 } & XXIV & 6,19 \\
\hline \multirow{2}{*}{60} & XXX & $-3,74$ \\
\cline { 2 - 3 } & X & 5,85 \\
\hline & I & $-5,68$ \\
\cline { 2 - 3 } & X & 5,30 \\
\hline
\end{tabular}


Se repitió el razonamiento para el cemento CPF40, y analizando todas las series, se obtuvieron las mayores desviaciones individuales según los días de rotura (no se muestra la tabla).

Según los resultados obtenidos, por ejemplo, a los 3 días, la desviación máxima negativa, se encontró en la serie XX con un -23,03\% de desviación. Lo que significa que esta serie rompió a los 28 días un $23,03 \%$ por debajo del valor teórico que debía dar aplicando la fórmula.

La desviación máxima positiva, le correspondió a la serie VI, con un $+34,35 \%$ de desviación. Es decir que esta serie a los 28 días rompió un 34,35\% arriba del valor teórico que debía dar aplicando la fórmula. De todos estos valores, cómo ya se expresó, las desviaciones más peligrosas son las "negativas", ya que significa que, en la realidad, obtenemos valores menores a los 28 días, de los teóricos calculados. Los valores, para este caso, se muestran en la Tabla 3.

Tabla 3. Desviaciones individuales, máximas y mínimas para mezclas con cemento CPF 40.

\begin{tabular}{|c|c|c|}
\hline Días de rotura & Serie & Desviación en \%. Máximas y mínimas \\
\hline \multirow{2}{*}{3,00} & XX & $-23,03$ \\
\cline { 2 - 3 } & VI & 34,35 \\
\hline \multirow{2}{*}{7,00} & XXIII & $-9,63$ \\
\cline { 2 - 3 } & V & 17,16 \\
\hline \multirow{2}{*}{14,00} & XVIII & $-7,00$ \\
\cline { 2 - 3 } & V & 8,20 \\
\hline \multirow{2}{*}{45,00} & I & $-8,50$ \\
\cline { 2 - 3 } & XIII & 3,08 \\
\hline \multirow{2}{*}{60,00} & III & $-10,54$ \\
\cline { 2 - 3 } & XVIII & 2,52 \\
\hline
\end{tabular}

\subsection{Estadística grupal}

El análisis individual, se realizó considerando ensayos individuales, siendo cada ensayo individual, el promedio de dos ensayos y que ninguno de ellos supere el 15\% de diferencia para considerar válido el valor.

Este análisis, es válido solo a los fines académicos, pero es antirreglamentario, ya que las normas solicitan un mínimo de tres valores individuales para determinar las tensiones características.

Para resolver esto se desarrolló un software que resuelva todas las combinaciones posibles de las treinta series tomados de a tres. Calcula las tensiones medias y las características al día de rotura establecido y a los veintiocho días, y calcula las diferencias en porcentaje entre el valor teórico de rotura y el valor real.

Esto, permitió encontrar las mayores desviaciones que un profesional podría encontrar al tomar tres valores característicos para un día determinado de ensayo y proyectarlo a los 28 días.

En las Tablas 4 y 5 se resume este concepto.

Se observa que, para tres días, en el caso de usarse cemento CPC40, se encuentran diferencia de hasta un $-35,55 \%$.

Para los 7 días la diferencia es de un $-15,74 \%$.

Para los 14, 45 y 60 días, las diferencias rondan el entre el 4\% y 7\%. Estos valores se muestran en Tabla 4.

Y para el caso de usar cemento CPF40, también analizando a tres días, se encontraron diferencia de hasta un $-39,57 \%$.

Para los 7 días la diferencia fue de un $-17,76 \%$. 
Para los 14, 45 y 60 días, las diferencias rondan el entre el 4\% y 10\%. Valores que se muestran en la Tabla 5.

Tabla 4. Desviaciones máximas, negativas y positivas de tres series para mezclas con cemento CPC 40.

\begin{tabular}{|c|c|}
\hline $\begin{array}{c}\text { Días de } \\
\text { rotura }\end{array}$ & Diferencia en porcentaje \\
\hline \multirow{2}{*}{3} & $-35,55$ \\
\cline { 2 - 2 } & 25,94 \\
\hline \multirow{2}{*}{7} & $-15,74$ \\
\cline { 2 - 2 } & 11,73 \\
\hline \multirow{2}{*}{14} & $-5,24$ \\
\hline \multirow{2}{*}{45} & 6,66 \\
\cline { 2 - 2 } & $-5,68$ \\
\hline \multirow{2}{*}{60} & $-4,32$ \\
\cline { 2 - 2 } & $-4,15$ \\
\hline
\end{tabular}

Tabla 5. Desviaciones máximas, negativas y positivas de tres series para mezclas con cemento CPF 40.

\begin{tabular}{|c|c|}
\hline \multirow{2}{*}{$\begin{array}{c}\text { Días de } \\
\text { rotura }\end{array}$} & Diferencia en porcentaje \\
\hline \multirow{2}{*}{3} & $-23,66$ \\
\cline { 2 - 2 } & 39,57 \\
\hline \multirow{2}{*}{7} & $-9,48$ \\
\cline { 2 - 2 } & 17,76 \\
\hline \multirow{2}{*}{14} & $-4,6$ \\
\cline { 2 - 2 } & 9,99 \\
\hline \multirow{2}{*}{45} & $-6,55$ \\
\cline { 2 - 2 } & 3,52 \\
\hline \multirow{2}{*}{60} & $-6,5$ \\
\cline { 2 - 2 } & 3,21 \\
\hline
\end{tabular}

\section{RESULTADOS}

Estos resultados indicaron que pretender ensayar probetas a los tres días, con un mínimo de tres series como pide el reglamento y proyectar su resistencia a los 28 días, es riesgoso según la importancia de la obra en cuestión ya que puede haber desviaciones de hasta un $-35,55 \%$.

A partir de los 7 días, las diferencias que puedan llegar a dar no son tan significativas y se podrá realizar la proyección, considerando siempre estas posibles diferencias.

A medida que el número de ensayos se incremente y se acerquen a treinta, las diferencias se acercarán a cero. 
En la práctica y en función de la importancia de la obra o de la parte de la obra que se deba realizar un control de calidad del hormigón, se aceptan desviaciones entre las tensiones características proyectadas aplicando la fórmula propuesta y los valores reales encontrados en los ensayos.

Por ejemplo, si se está haciendo el control de calidad del hormigón, de las columnas de planta baja de un edificio de 100 pisos, es evidente que se tomarán mínimos de valores de 30 series, a fin de lograr desviaciones insignificantes.

Si se está haciendo el control de calidad del hormigón de una losa de ese mismo edificio, se puede aceptar un $15 \%$ o un $10 \%$ de desvío.

En última instancia, es el propio proyectista el que impone esos límites en función de la importancia de la obra y de los cálculos realizados.

En general, la mayoría de los autores, admiten hasta un $15 \%$ de desvío.

Debido a ello, se introducen dos conceptos nuevos, que ayudaron a comprender el problema.

\section{1. Índice de Confianza}

Como vimos anteriormente, si ensayamos tres series a los tres días y proyectamos la resistencia teórica a los 28 días, puede haber desviaciones de hasta un 35,55\% en nuestro estudio.

La cantidad de combinaciones posibles es

$\mathrm{C}=\mathrm{n} ! /[\mathrm{r} ! *(\mathrm{n}-\mathrm{r}) !]$.

Siendo:

$\mathrm{n}=$ número de series ensayadas (30)

$\mathrm{r}=$ número de series parciales (3)

$\mathrm{C}=30$ ! / [3! * (30-3)! $]=4060$ combinaciones.

Esto indica que una de estas 4060 combinaciones posibles es la que determina el mayor desvío posible. La posibilidad de que ello ocurra, es:

$\%=1 / 4060=0,00246=0,246 \%$.

Se tiene entonces el $0,246 \%$ de probabilidades de que ocurra dicho evento, pero en algún momento puede ocurrir.

Se llama Índice de confianza, al porcentaje de probabilidades de que una desviación, en el control de calidad de un hormigón ocurra.

Este Índice depende de la cantidad de series ensayadas y del día de rotura.

Para ello se desarrolló un programa de software que analiza estas situaciones.

Este programa, realiza para cada edad en que se ensayan las probetas $(3,7,14,28,45$ y 60), todas las combinaciones posibles de las 30 series tomadas de a tres, cuatro, cinco, etc.

Para cada una de ellas, determina la tensión característica o especificada del día de ensayo y a los 28 días.

Establece la tensión teórica a los 28 días proyectada aplicando la formula y finalmente determina las diferencias entre esos valores teóricos proyectados y los realmente encontrados con los ensayos, tanto en valores como en porcentajes.

En las Tabla 6 y 7 se resume este concepto.

Se toman los ensayos con roturas a los tres días.

Para el caso de mezclas usando CPC40, mostrado en la Tabla 6, la probabilidad de que la desviación tomando combinaciones de a tres probetas, esté por debajo del $10 \%$ es de solo un $24,31 \%$, que esté por debajo del $20 \%$ es del $68 \%$ y por último que esté por debajo del $30 \%$ es aproximadamente del $94 \%$ $\mathrm{Si}$ estamos controlando la calidad de un hormigón y ensayamos probetas de tres series y proyectamos la resistencia característica a los 28 días, podemos determinar qué posibilidades existen de que la desviación supere el 15\%, o sea que se puede determinar la CONFIANZA, de que dicho porcentaje de desvío no sea superado. 
Tabla 6. Índice de confianza para rotura al tercer día y combinaciones de tres series para CPC 40.

\begin{tabular}{|c|c|}
\hline \multicolumn{2}{|c|}{$\begin{array}{c}\text { Combinación de 3 } \\
\text { probetas (4060) }\end{array}$} \\
\hline Diferencia & $\begin{array}{c}\text { Índice } \\
\text { confianza }\end{array}$ \\
\hline 10 & 24,31 \\
\hline 20 & 68 \\
\hline 30 & 93,69 \\
\hline
\end{tabular}

Y en el caso de mezclas usando CPF40, los valores obtenidos se muestran en la Tabla 7.

Tabla 7. Índice de confianza para rotura al tercer día y combinaciones de tres series para CPF 40.

\begin{tabular}{|c|c|}
\hline \multicolumn{2}{|c|}{$\begin{array}{c}\text { Combinación de 3 } \\
\text { probetas (4060) }\end{array}$} \\
\hline Diferencia & $\begin{array}{c}\text { Índice } \\
\text { confianza }\end{array}$ \\
\hline 10 & 22,20 \\
\hline 20 & 65,9 \\
\hline 30 & 91,69 \\
\hline
\end{tabular}

\section{2. Índice de certeza}

El Índice de certeza, Es el número mínimo de series a ensayar, de tal manera de asegurar una desviación máxima determinada.

Esta desviación máxima, está definida por el lote que se está controlando.

Se acepta un máximo de $15 \%$, pero varía según lo explicado en 3.3.

Para ello, se recurre nuevamente al software desarrollado.

Para cada edad de ensayo $(3,7,14,28,45$, y 60 días), se realizan todas las combinaciones posibles de treinta elementos tomados de a tres y se determina la serie de mayor desvío.

Si ese desvío es mayor del aceptado, se sigue combinando treinta elementos tomados de a cuatro y así sucesivamente, hasta encontrar un desvío que sea menor al permitido.

Ese número mínimo se series a ensayar, de tal manera de obtener una desviación máxima establecida previamente, se llama Índice de certeza.

Si tomamos como índice de desviación máximo el 15\%, el problema principal se registra entre el día tres y el día seis, ya que a los siete días las desviaciones máximas obtenidas tomando un mínimo de tres series, está dentro de lo previsto.

Los resultados para los ensayos el día tres, están resumidos en la Tabla 8 para mezclas usando cemento CPC40 y Tabla 9 para el cemento CPF40.

En la primer columna, se indica el número de series tomadas para hacer todas las combinaciones posibles.

La segunda columna, indica la máxima desviación encontrada para una serie, a tres días de ensayo. 
Tabla 8. Índice de certeza para cemento CPC 40 a tres días de rotura y 15\% de desviación máxima

\begin{tabular}{|c|c|c|}
\hline Número de series & Desviación en \% (-) & Índice de certeza \\
\hline 3 & $-35,55$ & \\
\hline 4 & $-28,98$ & \\
\hline 5 & $-24,18$ & \multirow{2}{*}{8} \\
\hline 6 & $-19,42$ & \\
\hline 7 & $-16,00$ & \\
\hline 8 & $-10,94$ & \\
\hline
\end{tabular}

Para el caso de usarse cemento CPC40, se observa que al tomar 8 series, se obtuvo una desviación máxima del 10,94\%, menor al $15 \%$ permitido.

Esto indica que si se quiere asegurar que la desviación máxima no sobrepase el 15\%, al ensayar a los tres días y proyectar la resistencia a los 28 días, se deberán tomar como mínimo ocho series de ensayo, lo que significa tomar muestras de ocho pastones de hormigón. El Índice de certeza es ocho.

Tabla 9. Índice de certeza para cemento CPF 40 a tres días de rotura y 15\% de desviación máxima

\begin{tabular}{|c|c|c|}
\hline Número de series & Desviación en \% (+) & Índice de certeza \\
\hline 3 & 46,71 & \\
\hline 4 & 42,71 & \\
\hline 5 & 37,78 & \\
\hline 6 & 32,41 & \multirow{2}{*}{9} \\
\hline 7 & 26,26 & \\
\hline 8 & 19,68 & \\
\hline 9 & 14,30 & \\
\hline
\end{tabular}

Para el caso de mezclas usando CPF40, se observa que al tomar 9 series, se obtiene una desviación máxima del 14,30\%, menor al $15 \%$ permitido.

Esto indica que si se quiere asegurar que la desviación máxima no sobrepase el 15\%, al ensayar a los tres días y proyectar la resistencia a los 28 días, se deberá tomar como mínimo nueve series de ensayo, lo que significa tomar muestras de nueve pastones de hormigón. El Índice de certeza para este caso es nueve.

\section{CONCLUSIONES}

En particular se ha comprobado, que a medida que disminuye la edad en que se ensaya el hormigón, aumentan las dispersiones, al predecir la resistencia a los 28 días, utilizando las fórmulas propuestas en el trabajo desarrollado para el proyecto "Evoluación de la resistencia del hormigón en el tiempo usando cementos CPC 40 y CPF 40 con áridos de la zona" (Schierloh et.al., 2017 -2018). Con este trabajo se pudo comprobar que la dispersión encontrada, entre resultados de ensayos de compresión a edades menores a 7 días y la predicción de la resistencia característica o especificada a los 28 días, cuando las series son las mínimas exigidas por norma (3), aumentan provocando incertidumbre. En consecuencia, se concluye que solo es posible predecir la resistencia característica o especificada a los 28 días, partiendo de ensayos en probetas realizados a edades iguales o mayores a 7 días, para que el rango de dispersiones se encuentre dentro de los parámetros admisibles (menores al 15\%), empleando 
el método tradicional de curado en cámara húmeda y para cantidades de series mínimas (3) exigidas en el país.

Para rangos de tiempo comprendidos entre los 3 días y los 7 días, se debe aumentar el número de series o pastones a ensayar, a fin de obtener dispersiones aceptables, lo que se definió en este trabajo como "Índice de certeza". Este Índice, es la cantidad mínima de pastones o series a ensayar, que en el caso del cemento CPC 40 resulto ser ocho y en el caso de cementos CPF 40 nueve.

Esto se debió fundamentalmente a diferentes factores:

a- Variación en la composición química de los cementos fabricados en Argentina, para el caso de estudio.

b- Falta de uniformidad en los horarios de comienzo de las reacciones químicas de los componentes del cemento (alitas, belitas, etc).

c- Falta de uniformidad en el porcentaje de combinaciones de los componentes químicos del cemento, a medida que transcurre el tiempo.

\section{AGRADECIMIENTOS}

Los autores desean agradecer en primer lugar a las autoridades de la Facultad Regional Concepción del Uruguay de la Universidad Tecnológica Nacional, por su apoyo sin el cual no se hubiese podido desarrollar esta investigación, a las empresas Hosifa Constructora S.A., Traza S.A., JCB Construcciones y Lambert Hermanos, por el aporte en equipamientos y en materiales imprescindibles para este trabajo. Y muy especialmente al Ingeniero Héctor Retamal por su asesoramiento continuo, aporte de áridos y planta hormigonera.

\section{REFERENCIAS}

Centro de Investigación de los Reglamentos Nacionales de Seguridad para las Obras Civiles. (2005). CIRSOC 201. Reglamento argentino de estructuras de hormigón. Argentina.

Comisión Permanente del Hormigón, EHE (2008), “Instrucción de Hormigón Estructural. Ministério de obras públicas e urbanismo". Madrid, España.

FAVA A.S.C. (1948), "Resistencia de rotura a la compresión en función de la relación agua-cemento y la edad", Instituto del Cemento Portland Argentina (ICPA), Argentina.

HUMMEL H. (1966), "Prontuario del Hormigón”. Editorial Técnicos Asociados.

Schierloh, MI et al. (2017), "Estudio sobre variación de la resistencia a compresión en el tiempo, para hormigones utilizando cemento cpc40 y áridos de la zona de Concepción del Uruguay - Entre Ríos". Anales CONPAT2017, Asunción de Paraguay.

Schierloh, MI, Souchetti, RF, Deusich, LD, Alza, LJ (2018), "Estudio sobre variación de la resistencia a compresión en el tiempo, para hormigones utilizando cementos argentinos y áridos de la zona de Concepción del Uruguay - Entre Ríos”. Anales 22 Reunión Técnica AATH. Olavarría. Argentina.

Vialidad Nacional Argentina, "Pliego de Especificaciones Técnicas Particular - Control del Hormigones para Obras de Arte Mayores-Vialidad Nacional Argentina". Available: http://www.vialidad.gov.ar/puentes. [Citado 10 marzo de 2015]. 